\title{
Multidimensional quadratic and subquadratic BSDEs with special structure
}

\author{
Patrick Cheridito \\ Princeton University \\ Princeton, NJ 08544, USA
}

\author{
Kihun Nam \\ Princeton University \\ Princeton, NJ 08544, USA
}

January 2015

\begin{abstract}
We study multidimensional BSDEs of the form

$$
Y_{t}=\xi+\int_{t}^{T} f\left(s, Y_{s}, Z_{s}\right) d s-\int_{t}^{T} Z_{s} d W_{s}
$$

with bounded terminal conditions $\xi$ and drivers $f$ that grow at most quadratically in $Z_{s}$. We consider three different cases. In the first one the BSDE is Markovian, and a solution can be obtained from a solution to a related FBSDE. In the second case, the BSDE becomes a one-dimensional quadratic BSDE when projected to a one-dimensional subspace, and a solution can be derived from a solution of the one-dimensional equation. In the third case, the growth of the driver $f$ in $Z_{s}$ is strictly subquadratic, and the existence and uniqueness of a solution can be shown by first solving the BSDE on a short time interval and then extending it recursively.
\end{abstract}

Key words: Multidimensional backward stochastic differential equations, forward-backward stochastic differential equations, quadratic BSDEs, projectable BSDEs, strictly subquadratic BSDEs.

\section{Introduction}

We study multidimensional BSDEs of the form

$$
Y_{t}=\xi+\int_{t}^{T} f\left(s, Y_{s}, Z_{s}\right) d s-\int_{t}^{T} Z_{s} d W_{s}
$$

where $Y_{t}$ takes values in $\mathbb{R}^{d}, Z_{t}$ is $\mathbb{R}^{d \times n}$-valued and $W$ is an $n$-dimensional Brownian motion. If the terminal condition $\xi$ is square-integrable and the driver $f(t, y, z)$ Lipschitz continuous in 
$(y, z)$, the existence of a unique solution can be shown with a Picard-Lindelöf iteration argument, see e.g. Pardoux and Peng (1990) or El Karoui et al. (1997). Kobylanski (2000) proved that one-dimensional $(d=1)$ BSDEs with drivers of quadratic growth in $z$ have solutions if $\xi$ is bounded. Moreover, if $\xi$ has bounded Malliavin derivative, the growth of $f(s, y, z)$ in $z$ can be arbitrary; see Cheridito and Nam (2014). For multidimensional BSDEs the situation is more complicated because one cannot use comparison results; see e.g. Hu and Peng (2006). In fact, multidimensional BSDEs with drivers of quadratic growth in $z$ do not always admit solutions even if the terminal condition $\xi$ is bounded; see Frei and dos Reis (2011) for an example. An early result for superlinear multidimensional BSDEs was given by Bahlali et al. (2001), which assumed that the growth of $f(s, y, z)$ in $z$ is of the order $|z| \sqrt{\log |z|}$. It was generalized by Bahlali et al. (2010) to the case where $f(s, y, z)$ has strictly subquadratic growth in $z$ and satisfies a monotonicity condition. Tevzadze (2008) gave an existence and uniqueness result for multidimensional BSDEs with general drivers of quadratic $z$-growth in the case where the terminal condition has small $L^{\infty}$-norm.

In this paper we put restrictions on the driver. Three different cases are considered. In all three we assume $\xi$ to be bounded and use BMO-martingale theory together with Girsanov's theorem to construct an equivalent probability measure that can be used to prove the existence of a solution.

In Section 2 we assume the BSDE to be Markovian and related to an FBSDE of the form

$$
\begin{aligned}
d P_{t} & =G\left(t, P_{t}, Q_{t}, R_{t}\right) d t+d W_{t}, \quad P_{0}=0 \\
d Q_{t} & =-F\left(t, P_{t}, Q_{t}, R_{t}\right) d t+R_{t} d W_{t}, \quad Q_{T}=h\left(P_{T}\right)
\end{aligned}
$$

for a bounded function $h$. If the FBSDE has a solution, we change the probability measure to obtain a solution to a different FBSDE, from which a solution to the BSDE (1.1) can be derived. A similar approach was taken by Liang et al. (2010) but without proving that the solution is adapted to the filtration generated by the Brownian motion driving the BSDE; that is, they showed the existence of a weak solution. Here, we prove the existence of a strong solution. There exist different results in the literature guaranteeing the existence of a solution to (1.2). We use one of Delarue (2002) to derive that the BSDE (1.1) has a unique bounded solution. Mania and Schweizer (2005) and Ankirchner et al. (2009) studied the transformation of onedimensional quadratic BSDEs under a change of measure, but not with the aim of proving the existence of a classical solution. In Section 3 conditions are given under which equation (1.1) can be turned into a one-dimensional quadratic BSDE by projecting it on a one-dimensional subspace of $\mathbb{R}^{d}$. Results of Kobylanski (2000) guarantee that the one-dimensional equation has a solution. From there a solution to the multidimensional equation can be obtained by changing the probability measure and solving a linear equation. In Section 4 the growth of $f(s, y, z)$ in $z$ is assumed to be strictly subquadratic. This makes it possible to prove the existence of a unique solution on a short time interval with a contraction argument. Under an additional structural assumption, the solution can be estimated by taking conditional expectation with respect to 
an equivalent probability measure. Then the short-time solution can be extended to a global solution.

\section{Notation:}

In the whole paper $T \in \mathbb{R}_{+}$is a finite time horizon and $\left(W_{t}\right)_{0 \leq t \leq T}$ an $n$-dimensional Brownian motion on a probability space $(\Omega, \mathcal{F}, \mathbb{P})$. By $\mathbb{F}$ we denote the augmented filtration $\left(\mathcal{F}_{t}\right)$ generated by $W$. The terminal condition $\xi$ is a bounded $d$-dimensional $\mathcal{F}_{T}$-measurable random vector and the driver $f:[0, T] \times \Omega \times \mathbb{R}^{d} \times \mathbb{R}^{d \times n} \rightarrow \mathbb{R}^{d}$ a $\mathcal{P} \otimes \mathcal{B}\left(\mathbb{R}^{d}\right) \otimes \mathcal{B}\left(\mathbb{R}^{d \times n}\right)$-measurable mapping, where $\mathcal{P}$ denotes the predictable sigma-algebra and $\mathcal{B}\left(\mathbb{R}^{d}\right)$ and $\mathcal{B}\left(\mathbb{R}^{d \times n}\right)$ the Borel sigma-algebras on $\mathbb{R}^{d}$ and $\mathbb{R}^{d \times n}$, respectively. As usual, we understand equalities and inequalities between random variables in the $\mathbb{P}$-almost sure sense. $Y, \xi$ and $f$ are understood as $d \times 1$-matrices, $W$ as an $n \times 1$-matrix and $Z$ as a $d \times n$-matrix. By $Z^{T}$ we denote the transpose of $Z$ and by $|\cdot|$ the Euclidean norm. That is, for a vector $Y,|Y|:=\sqrt{\sum_{i} Y_{i}^{2}}$, and for a matrix $Z,|Z|=\sqrt{\operatorname{tr}\left(Z Z^{T}\right)}$. $\mathbb{R}_{+}$denotes the set of nonnegative real numbers and $\|\cdot\|_{p}$ the $L^{p}$-norm. We need the following Banach spaces of stochastic processes:

- $\mathbb{S}^{p}\left(\mathbb{R}^{d}\right)$ : all $d$-dimensional continuous adapted processes satisfying

$$
\|Y\|_{\mathbb{S} p}:=\left\|\sup _{0 \leq t \leq T}\left|Y_{t}\right|\right\|_{p}<\infty
$$

- $\mathbb{H}^{p}\left(\mathbb{R}^{d \times n}\right)$ for $p<\infty$ : all $\mathbb{R}^{d \times n}$-valued predictable processes satisfying

$$
\|Z\|_{\mathbb{H}^{p}}:=\left\|\left(\int_{0}^{T}\left|Z_{s}\right|^{2} d s\right)^{1 / 2}\right\|_{p}<\infty
$$

- $\mathbb{H}^{\infty}\left(\mathbb{R}^{d \times n}\right)$ : all $\mathbb{R}^{d \times n}$-valued predictable processes satisfying

$$
\|Z\|_{\mathbb{H} \infty}:=\operatorname{ess~sup}_{(t, \omega)}\left|Z_{t}(\omega)\right|<\infty
$$

- $\mathbb{H}^{\mathrm{BMO}}\left(\mathbb{R}^{d \times n}\right)$ : all $Z \in \mathbb{H}^{2}\left(\mathbb{R}^{d \times n}\right)$ satisfying

$$
\|Z\|_{\mathrm{BMO}}:=\sup _{\tau \in \mathcal{T}}\left\|\mathbb{E}_{\tau} \int_{\tau}^{T}\left|Z_{s}\right|^{2} d s\right\|_{\infty}^{1 / 2}<\infty,
$$

where $\mathcal{T}$ denotes the set of all $[0, T]$-valued stopping times $\tau$ and $\mathbb{E}_{\tau}$ the conditional expectation with respect to $\mathcal{F}_{\tau}$. 
By $\mathbb{S}_{[a, b]}^{p}\left(\mathbb{R}^{d}\right), \mathbb{H}_{[a, b]}^{p}\left(\mathbb{R}^{d \times n}\right)$ and $\mathbb{H}_{[a, b]}^{\mathrm{BMO}}\left(\mathbb{R}^{d \times n}\right)$ we denote the same spaces if the processes have time indexes in $[a, b]$.

For $H \in \mathbb{H}^{\mathrm{BMO}}\left(\mathbb{R}^{n \times 1}\right), \int_{0}^{t} H_{s}^{T} d W_{s}$ is a BMO-martingale and

$$
\mathcal{E}_{t}^{H}:=\exp \left(\int_{0}^{t} H_{s}^{T} d W_{s}-\frac{1}{2} \int_{0}^{T}\left|H_{s}\right|^{2} d s\right)
$$

a martingale; see Kazamaki (1994). So one obtains from Girsanov's theorem that $\mathcal{E}_{T}^{H} \cdot \mathbb{P}$ defines an equivalent probability measure, under which $W_{t}-\int_{0}^{t} H_{s} d s$ is a Brownian motion. Moreover, every $Z \in \mathbb{H}^{\mathrm{BMO}}\left(\mathbb{R}^{d \times n}\right)$ with respect to $\mathbb{P}$ is also in $\mathbb{H}^{\mathrm{BMO}}\left(\mathbb{R}^{d \times n}\right)$ with respect to $\mathcal{E}_{T}^{H} \cdot \mathbb{P}$.

\section{Markovian quadratic BSDEs}

In this section we consider BSDEs of the form

$$
Y_{t}=h\left(W_{T}\right)+\int_{t}^{T}\left\{F\left(s, W_{s}, Y_{s}, Z_{s}\right)+Z_{s} G\left(s, W_{s}, Y_{s}, Z_{s}\right)\right\} d s-\int_{t}^{T} Z_{s} d W_{s}
$$

for measurable functions $h: \mathbb{R}^{n} \rightarrow \mathbb{R}^{d}, F:[0, T] \times \mathbb{R}^{n} \times \mathbb{R}^{d} \times \mathbb{R}^{d \times n} \rightarrow \mathbb{R}^{d}$ and $G:[0, T] \times \mathbb{R}^{n} \times \mathbb{R}^{d} \times$ $\mathbb{R}^{d \times n} \rightarrow \mathbb{R}^{n}$.

The following theorem gives conditions under which (2.1) has a solution if there is a solution to a related FBSDE.

Theorem 2.1. Assume that there exists a constant $C \in \mathbb{R}_{+}$and a nondecreasing function $\rho$ : $\mathbb{R}_{+} \rightarrow \mathbb{R}_{+}$such that the following conditions hold:

(A1) $|h(x)| \leq C$

(A2) $y^{T} F(t, x, y, z) \leq C|y|(1+|y|+|z|)$ for all $(t, x, y, z) \in[0, T] \times \mathbb{R}^{n} \times \mathbb{R}^{d} \times \mathbb{R}^{d \times n}$

(A3) $|G(t, x, y, z)| \leq \rho(|y|)(1+|z|)$ for all $(t, y, z) \in[0, T] \times \mathbb{R}^{d} \times \mathbb{R}^{d \times n}$

(A4) The FBSDE

$$
\begin{aligned}
d P_{t} & =G\left(t, P_{t}, Q_{t}, R_{t}\right) d t+d W_{t}, \quad P_{0}=0 \\
d Q_{t} & =-F\left(t, P_{t}, Q_{t}, R_{t}\right) d t+R_{t} d W_{t}, \quad Q_{T}=h\left(P_{T}\right)
\end{aligned}
$$

has a solution $(P, Q, R) \in \mathbb{H}^{2}\left(\mathbb{R}^{n}\right) \times \mathbb{S}^{2}\left(\mathbb{R}^{d}\right) \times \mathbb{H}^{2}\left(\mathbb{R}^{d \times n}\right)$ such that $Q_{t}=q(t, P)$ and $R_{t}=r(t, P)$ for predictable functions $q:[0, T] \times C\left([0, T], \mathbb{R}^{n}\right) \rightarrow \mathbb{R}^{d}$ and $r:[0, T] \times C\left([0, T], \mathbb{R}^{n}\right) \rightarrow \mathbb{R}^{d \times n}$.

Then $\left(Y_{t}, Z_{t}\right)=(q(t, W), r(t, W))$ is a solution of the BSDE (2.1) in $\mathbb{S}^{\infty}\left(\mathbb{R}^{d}\right) \times \mathbb{H}^{\mathrm{BMO}}\left(\mathbb{R}^{d \times n}\right)$, and $Z$ is bounded if $R$ is bounded. 
Proof. One obtains from Itô's formula that for every $a \in \mathbb{R}_{+}$and $[0, T]$-valued stopping time $\tau$,

$$
e^{a \tau}\left|Q_{\tau}\right|^{2}=e^{a T}\left|h\left(P_{T}\right)\right|^{2}+\int_{\tau}^{T} e^{a s}\left(2 Q_{s}^{T} F\left(s, P_{s}, Q_{s}, R_{s}\right)-\left|R_{s}\right|^{2}-a\left|Q_{s}\right|^{2}\right) d s-\int_{\tau}^{T} 2 e^{a s} Q_{s}^{T} R_{s} d W_{s} .
$$

Since $Q \in \mathbb{S}^{2}\left(\mathbb{R}^{d}\right)$ and $R \in \mathbb{H}^{2}\left(\mathbb{R}^{d \times n}\right)$, one has

$$
\mathbb{E} \sqrt{\int_{0}^{T}\left|Q_{s}^{T} R_{s}\right|^{2} d s} \leq \mathbb{E} \sup _{0 \leq s \leq T}\left|Q_{s}\right| \sqrt{\int_{0}^{T}\left|R_{s}\right|^{2} d s} \leq\|Q\|_{\mathbb{S}^{2}}\|R\|_{\mathbb{H}^{2}}<\infty .
$$

So it follows from the Burkholder-Davis-Gundy inequality that $\sup _{0 \leq t \leq T}\left|\int_{0}^{t} 2 e^{a s} Q_{s}^{T} R_{s} d W_{s}\right|$ is integrable, implying that the local martingale $\int_{0}^{t} 2 e^{a s} Q_{s}^{T} R_{s} d W_{s}$ is a true martingale. Therefore,

$$
e^{a \tau}\left|Q_{\tau}\right|^{2}=\mathbb{E}_{\tau}\left(e^{a T}\left|h\left(P_{T}\right)\right|^{2}+\int_{\tau}^{T} e^{a s}\left(2 Q_{s}^{T} F\left(s, P_{s}, Q_{s}, R_{s}\right)-\left|R_{s}\right|^{2}-a\left|Q_{s}\right|^{2}\right) d s\right) .
$$

By assumption (A2),

$$
\begin{aligned}
& 2 Q_{s}^{T} F\left(s, P_{s}, Q_{s}, R_{s}\right)-\left|R_{s}\right|^{2}-a\left|Q_{s}\right|^{2} \leq 2 C\left|Q_{s}\right|\left(1+\left|Q_{s}\right|+\left|R_{s}\right|\right)-\left|R_{s}\right|^{2}-a\left|Q_{s}\right|^{2} \\
& \leq C^{2}+\left(2 C^{2}+2 C+1-a\right)\left|Q_{s}\right|^{2}-\frac{1}{2}\left|R_{s}\right|^{2} .
\end{aligned}
$$

So for $a=2 C^{2}+2 C+1$, one obtains

$$
\begin{aligned}
& \left|Q_{\tau}\right|^{2}+\frac{1}{2} \mathbb{E}_{\tau} \int_{\tau}^{T}\left|R_{s}\right|^{2} d s \leq e^{a \tau}\left|Q_{\tau}\right|^{2}+\frac{1}{2} \mathbb{E}_{\tau} \int_{\tau}^{T} e^{a s}\left|R_{s}\right|^{2} d s \\
& \leq \mathbb{E}_{\tau}\left(e^{a T}\left|h\left(P_{T}\right)\right|^{2}+C^{2} \int_{\tau}^{T} e^{a s} d s\right) \leq C^{2} e^{a T}(1+T) .
\end{aligned}
$$

In particular, $Q$ is in $\mathbb{S}^{\infty}\left(\mathbb{R}^{d}\right)$ and $R$ in $\mathbb{H}^{\mathrm{BMO}}\left(\mathbb{R}^{d \times n}\right)$. By assumption (A3), one has

$$
\left|G\left(s, P_{s}, Q_{s}, R_{s}\right)\right| \leq \rho\left(C^{2} e^{a T}(1+T)\right)\left(1+\left|R_{s}\right|\right),
$$

from which it follows that $G\left(s, P_{s}, Q_{s}, R_{s}\right)$ belongs to $\mathbb{H}^{\mathrm{BMO}}\left(\mathbb{R}^{n \times 1}\right)$. Therefore, $P$ is a Brownian motion under the measure $\mathcal{E}_{T}^{-G} \cdot \mathbb{P}$, and $R$ is still in $\mathbb{H}^{\mathrm{BMO}}\left(\mathbb{R}^{d \times n}\right)$ under $\mathcal{E}_{T}^{-G} \cdot \mathbb{P}$. The backward equation in (A4) can be written as

$$
d Q_{t}=-\left(F\left(t, P_{t}, Q_{t}, R_{t}\right)+R_{t} G\left(t, P_{t}, Q_{t}, R_{t}\right)\right) d t+R_{t} d P_{t}, \quad Q_{T}=h\left(P_{T}\right) .
$$

But since $Q_{t}=q(t, P)$ and $R_{t}=r(t, P)$, one has

$$
d q(t, P)=-\left(F\left(t, P_{t}, q(t, P), r(t, P)\right)+r(t, P) G\left(t, P_{t}, q(t, P), r(t, P)\right)\right) d t+r(t, P) d P_{t} .
$$

So $(Y, Z)=(q(\cdot, W), r(\cdot, W))$ is in $\mathbb{S}^{\infty}\left(\mathbb{R}^{d}\right) \times \mathbb{H}^{\mathrm{BMO}}\left(\mathbb{R}^{d \times n}\right)$ and satisfies

$$
d Y_{t}=-\left(F\left(t, W_{t}, Y_{t}, Z_{t}\right)+Z_{t} G\left(t, W_{t}, Y_{t}, Z_{t}\right)\right) d t+Z_{t} d W_{t}, \quad Y_{T}=h\left(W_{T}\right) .
$$

Finally, if $R$ is bounded, then so is $Z$. 
Remark 2.2. Since the BSDE (2.1) is Markovian, it is related to the semilinear parabolic PDE with terminal condition

$$
u_{t}+\frac{1}{2} \triangle u+F(t, x, u, \nabla u)+(\nabla u) g(t, x, u, \nabla u)=0, \quad u(T, x)=h(x) .
$$

For example, if the PDE has a $C^{1,2}$-solution $u:[0, T] \times \mathbb{R}^{n} \rightarrow \mathbb{R}^{d}$, it follows from Itô's formula that $\left(Y_{t}, Z_{t}\right)=\left(u\left(t, W_{t}\right), \nabla u\left(t, W_{t}\right)\right)$ solves the BSDE (2.1). But the standard construction of a viscosity solution to the PDE from a BSDE solution does not work because the necessary comparison results do not extend from the one- to the multidimensional case; see Peng (1999).

A crucial assumption of Theorem 2.1 is (A4). There exist different results in the FBSDE literature from which it follows. The following proposition derives the existence of a unique solution to the quadratic BSDE (2.1) from an FBSDE result of Delarue (2002).

Proposition 2.3. Assume there exists a constant $C \in \mathbb{R}_{+}$such that for all $t, x, x^{\prime}, y, y^{\prime}, z, z^{\prime}$ the following hold:

$$
\begin{aligned}
& \text { - }\left|F(t, x, y, z)-F\left(t, x^{\prime}, y^{\prime}, z^{\prime}\right)\right| \leq C\left(\left|x-x^{\prime}\right|+\left|y-y^{\prime}\right|+\left|z-z^{\prime}\right|\right) \\
& \text { - }\left|G(t, x, y, z)-G\left(t, x^{\prime}, y^{\prime}, z^{\prime}\right)\right| \leq C\left(\left|x-x^{\prime}\right|+\left|y-y^{\prime}\right|+\left|z-z^{\prime}\right|\right) \\
& \text { - }\left|h(x)-h\left(x^{\prime}\right)\right| \leq C\left|x-x^{\prime}\right| \\
& \text { - }|F(t, x, 0,0)|+|G(t, x, 0,0)+| h(x) \mid \leq C .
\end{aligned}
$$

Then the BSDE (2.1) has a unique solution $(Y, Z)$ in $\mathbb{S}^{\infty}\left(\mathbb{R}^{d}\right) \times \mathbb{H}^{\infty}\left(\mathbb{R}^{d \times n}\right)$, and it is of the form $Y_{t}=y\left(t, W_{t}\right), Z_{t}=\nabla_{x} y\left(t, W_{t}\right)$, where $y:[0, T] \times \mathbb{R}^{n} \rightarrow \mathbb{R}^{d}$ is a continuous function that is uniformly Lipschitz in $x \in \mathbb{R}^{n}$ and $\nabla_{x}$ denotes the weak derivative with respect to $x$ in the Sobolev sense.

Proof. It follows from the assumptions that the conditions of Proposition 2.4 and Theorem 2.6 of Delarue (2002) hold. Therefore, the FBSDE in (A4) of Theorem 2.1 has a unique solution $(P, Q, R)$ in $\mathbb{H}^{2}\left(\mathbb{R}^{n}\right) \times \mathbb{S}^{\infty}\left(\mathbb{R}^{d}\right) \times \mathbb{H}^{\infty}\left(\mathbb{R}^{d \times n}\right)$ such that $Q$ is of the form $Q_{t}=q\left(t, P_{t}\right)$ for a bounded continuous function $q:[0, T] \times \mathbb{R}^{n} \rightarrow \mathbb{R}^{d}$ which is uniformly Lipschitz in $x \in \mathbb{R}^{n}$. As a consequence, the process $G_{t}=G\left(t, P_{t}, Q_{t}, R_{t}\right)$ is bounded, from which it follows that $\mathcal{E}_{T}^{-G} \cdot \mathbb{P}$ defines a probability measure equivalent to $\mathbb{P}$, under which $P$ is an $\mathbb{F}$-adapted $n$-dimensional Brownian motion. It can be seen from the representation

$$
Q_{t}=q\left(t, P_{t}\right)=Q_{0}-\int_{0}^{t}\left\{F\left(s, P_{s}, Q_{s}, R_{s}\right)+R_{s} G\left(s, P_{s}, Q_{s}, R_{s}\right)\right\} d s+\int_{0}^{t} R_{s} d P_{s}
$$

that $Q$ is a continuous $\mathbb{F}$-semimartingale. By Stricker's theorem, it is also a continuous semimartingale with respect to the filtration $\mathbb{F}^{P}$ generated by $P$. In particular, it has a unique canonical $\left(\mathbb{F}^{P}, \tilde{\mathbb{P}}\right)$-semimartingale decomposition $Q_{t}=Q_{0}+M_{t}+A_{t}$, where $M$ is a continuous 
$\left(\mathbb{F}^{P}, \tilde{\mathbb{P}}\right)$-local martingale and $A$ a finite variation process with $M_{0}=A_{0}=0$. By the martingale representation theorem, $M_{t}$ can be written as $M_{t}=\int_{0}^{t} H_{s} d P_{s}$ for a unique $\mathbb{F}^{P}$-predictable process $H$. But since $P$ is an $\mathbb{F}$-Brownian motion under $\tilde{\mathbb{P}}, Q_{t}=Q_{0}+M_{t}+A_{t}$ is also the unique canonical $(\mathbb{F}, \tilde{\mathbb{P}})$-semimartingale decomposition of $Q$. It follows that $R=H$, and therefore, $R_{t}=r(t, P)$ for a predictable function $r:[0, T] \times C\left([0, T], \mathbb{R}^{n}\right) \rightarrow \mathbb{R}^{d \times n}$. This shows that (A4) holds. Since (A1)-(A3) of Theorem 2.1 are satisfied as well, one obtains that $\left(Y_{t}, Z_{t}\right)=\left(q\left(t, W_{t}\right), r(t, W)\right)$ is a solution of the BSDE (2.1). Moreover, since $q$ is continuous and $q\left(t, P_{t}\right)$ an Itô process, it follows from Theorem 1 of Chitashvili and Mania (1996) that $r(t, P)=\nabla_{x} q\left(t, P_{t}\right)$, where $\nabla_{x} q$ is a bounded weak derivative of $q$ with respect to $x$ in the Sobolev sense. This shows that $\left(q\left(t, W_{t}\right), \nabla_{x} q\left(t, W_{t}\right)\right)$ is a solution of (2.1) in $\mathbb{S}^{\infty}\left(\mathbb{R}^{d}\right) \times \mathbb{H}^{\infty}\left(\mathbb{R}^{d \times n}\right)$.

Now assume $(\tilde{Y}, \tilde{Z})$ is another solution of (2.1) in $\mathbb{S}^{\infty}\left(\mathbb{R}^{d}\right) \times \mathbb{H}^{\infty}\left(\mathbb{R}^{d \times n}\right)$ and let $L$ be a common bound for $Y, Y^{\prime}, Z, Z^{\prime}$. Then $(Y, Z)$ and $\left(Y^{\prime}, Z^{\prime}\right)$ are both solutions of the modified BSDE

$$
Y_{t}=h\left(W_{T}\right)+\int_{t}^{T} f\left(s, W_{s}, \pi_{L}\left(Y_{s}, Z_{s}\right)\right) d s-\int_{t}^{T} Z_{s} d W_{s}
$$

where

$$
f(t, x, y, z):=F(t, x, y, z)+z G(t, x, y, z) \quad \text { and } \quad \pi_{L}(y, z):=(\min \{1, L /|y|\} y, \min \{1, L /|z|\} z) .
$$

Since this BSDE satisfies the conditions of Pardoux and Peng (1990), it has a unique solution in $\mathbb{S}^{\infty}\left(\mathbb{R}^{d}\right) \times \mathbb{H}^{\infty}\left(\mathbb{R}^{d \times n}\right)$, and it follows that $(Y, Z)=(\tilde{Y}, \tilde{Z})$.

Remark 2.4. The assumptions of Proposition 2.3 can be slightly relaxed such that the conditions of Theorem 2.6 of Delarue (2002) are still met. Then the same arguments yield the existence of a bounded solution $(Y, Z)$ to the BSDE (2.1). Uniqueness can be shown by using Pardoux (1999) instead of Pardoux and Peng (1990). Alternatively, the assumptions of Proposition 2.3 can be modified such that they imply some other FBSDE result, such as e.g., the one of Pardoux and Tang (1999).

\section{Projectable quadratic BSDEs}

Definition 3.1. We call a multidimensional BSDE projectable if its driver can be written as

$$
f(s, y, z)=P\left(s, a^{T} y, a^{T} z\right)+y Q\left(s, a^{T} y, a^{T} z\right)+z R\left(s, a^{T} y, a^{T} z\right)
$$

for a constant vector $a \in \mathbb{R}^{d}$ and predictable functions

$$
\begin{aligned}
& P:[0, T] \times \Omega \times \mathbb{R} \times \mathbb{R}^{1 \times n} \rightarrow \mathbb{R}^{d \times 1} \\
& Q:[0, T] \times \Omega \times \mathbb{R} \times \mathbb{R}^{1 \times n} \rightarrow \mathbb{R} \\
& R:[0, T] \times \Omega \times \mathbb{R} \times \mathbb{R}^{1 \times n} \rightarrow \mathbb{R}^{n \times 1} .
\end{aligned}
$$


A projectable BSDE becomes one-dimensional if projected on the line generated by $a \in \mathbb{R}^{d \times 1}$ :

$$
\begin{aligned}
a^{T} Y_{t}= & a^{T} \xi+\int_{t}^{T} a^{T}\left\{P\left(s, a^{T} Y_{s}, a^{T} Z_{s}\right)+Y_{s} Q\left(s, a^{T} Y_{s}, a^{T} Z_{s}\right)+Z_{s} R\left(s, a^{T} Y_{s}, a^{T} Z_{s}\right)\right\} d s \\
& -\int_{t}^{T} a^{T} Z_{s} d W_{s} .
\end{aligned}
$$

In the following theorem we consider a projectable BSDE under conditions ensuring that the projected BSDE has a solution. This makes it possible to derive the existence of a solution to the multidimensional BSDE.

Theorem 3.2. Consider a bounded terminal condition $\xi \in L^{\infty}\left(\mathcal{F}_{T}\right)^{d}$ and a driver $f$ of the form (3.1) such that

$$
|P(s, u, v)| \leq C(1+|u|), \quad|Q(s, u, v)| \leq C, \quad|R(s, u, v)| \leq C+\rho(|u|)|v|
$$

for a constant $C \in \mathbb{R}_{+}$and a nondecreasing function $\rho: \mathbb{R}_{+} \rightarrow \mathbb{R}_{+}$. Then the BSDE

$$
Y_{t}=\xi+\int_{t}^{T} f\left(s, Y_{s}, Z_{s}\right) d s-\int_{t}^{T} Z_{s} d W_{s}
$$

has a solution $(Y, Z) \in \mathbb{S}^{\infty}\left(\mathbb{R}^{d}\right) \times \mathbb{H}^{\mathrm{BMO}}\left(\mathbb{R}^{d \times n}\right)$.

Moreover, if

$$
F(s, u, v)=a^{T} P(s, u, v)+u Q(s, u, v)+v R(s, u, v)
$$

satisfies

$$
\left|F(s, u, v)-F\left(s, u^{\prime}, v^{\prime}\right) \leq C\right| u-u^{\prime}\left|+C\left(1+|v| \vee\left|v^{\prime}\right|\right)\right| v-v^{\prime} \mid,
$$

then (3.2) has only one solution $(Y, Z)$ in $\mathbb{S}^{\infty}\left(\mathbb{R}^{d}\right) \times \mathbb{H}^{\mathrm{BMO}}\left(\mathbb{R}^{d \times n}\right)$.

Proof. By Theorem 2.3 of Kobylanski (2000), the one-dimensional BSDE

$$
U_{t}=a^{T} \xi+\int_{t}^{T} F\left(s, U_{s}, V_{s}\right) d s-\int_{t}^{T} V_{s} d W_{s}
$$

has a solution $(U, V) \in \mathbb{S}^{\infty}(\mathbb{R}) \times \mathbb{H}^{2}\left(\mathbb{R}^{1 \times n}\right)$. Therefore, there exists a constant $K \geq 0$ such that $\left|F\left(s, U_{s}, V_{s}\right)\right| \leq K\left(1+\left|V_{s}\right|^{2}\right)$, and it follows like in the proof of Proposition 2.1 in Briand and Elie (2013) that $V$ is in $\mathbb{H}^{\mathrm{BMO}}\left(\mathbb{R}^{1 \times n}\right)$. Denote $P_{s}:=P\left(s, U_{s}, V_{s}\right), Q_{s}:=Q\left(s, U_{s}, V_{s}\right), R_{s}=R\left(s, U_{s}, V_{s}\right)$ and assume the multidimensional linear BSDE

$$
Y_{t}=\xi+\int_{t}^{T}\left(P_{s}+Y_{s} Q_{s}+Z_{s} R_{s}\right) d s-\int_{t}^{T} Z_{s} d W_{s}
$$


has a solution $(Y, Z) \in \mathbb{S}^{\infty}\left(\mathbb{R}^{d}\right) \times \mathbb{H}^{\mathrm{BMO}}\left(\mathbb{R}^{d \times n}\right)$. It follows from the assumptions that $\left|R_{s}\right| \leq$ $C+\rho\left(\|U\|_{\mathbb{S} \infty}\right)\left|V_{s}\right|$. So $R$ is in $\mathbb{H}^{\mathrm{BMO}}\left(\mathbb{R}^{n \times 1}\right)$, and $\tilde{\mathbb{P}}:=\mathcal{E}_{T}^{R} \cdot \mathbb{P}$ is an equivalent probability measure under which $\tilde{W}_{t}=W_{t}-\int_{0}^{t} R_{s} d s$ is a Brownian motion. Now one can write

$$
Y_{t}=\xi+\int_{t}^{T}\left(P_{s}+Y_{s} Q_{s}\right) d s-\int_{t}^{T} Z_{s} d \tilde{W}_{s}
$$

from which it follows that

$$
e^{\int_{0}^{t} Q_{u} d u} Y_{t}=e^{\int_{0}^{T} Q_{u} d u} \xi+\int_{t}^{T} e^{\int_{0}^{s} Q_{u} d u} P_{s} d s-\int_{t}^{T} e^{\int_{0}^{s} Q_{u} d u} Z_{s} d \tilde{W}_{s},
$$

and therefore,

$$
e^{\int_{0}^{t} Q_{s} d s} Y_{t}=\tilde{\mathbb{E}}_{t}\left[e^{\int_{0}^{T} Q_{u} d u} \xi+\int_{t}^{T} e^{\int_{0}^{s} Q_{u} d u} P_{s} d s\right],
$$

where $\tilde{\mathbb{E}}$ denotes expectation with respect to $\tilde{\mathbb{P}}$. This uniquely determines $Y$. Now $Z$ is uniquely given by (3.6). To show that (3.5) has a solution in $\mathbb{S}^{\infty}\left(\mathbb{R}^{d}\right) \times \mathbb{H}^{\mathrm{BMO}}\left(\mathbb{R}^{d \times n}\right)$, one can define $Y$ by (3.7), which is equivalent to

$$
\Gamma_{t} Y_{t}=\mathbb{E}_{t}\left[\Gamma_{T} \xi+\int_{t}^{T} \Gamma_{s} P_{s} d s\right]
$$

where $\Gamma$ is the unique solution of the SDE

$$
d \Gamma_{t}=\Gamma_{t}\left(Q_{s} d s+R_{s}^{T} d W_{s}\right), \quad \Gamma_{0}=1 .
$$

Then $Y$ belongs to $\mathbb{S}^{\infty}\left(\mathbb{R}^{d}\right)$, and by the martingale representation theorem, there exists a unique predictable process $Z$ such that $\Gamma Z$ belongs to $\mathbb{H}^{2}\left(\mathbb{R}^{d \times n}\right)$ and

$$
\int_{0}^{T} \Gamma_{s}\left(Y_{s} R_{s}^{T}+Z_{s}\right) d W_{s}=\Gamma_{T} \xi+\int_{0}^{T} \Gamma_{s} P_{s} d s-\mathbb{E}\left[\Gamma_{T} \xi+\int_{0}^{T} \Gamma_{s} P_{s} d s\right] .
$$

Since $Y_{0}=\mathbb{E}\left[\Gamma_{T} \xi+\int_{0}^{T} \Gamma_{s} P_{s} d s\right]$, one has

$$
Y_{0}+\int_{0}^{t} \Gamma_{s}\left(Y_{s} R_{s}^{T}+Z_{s}\right) d W_{s}=\mathbb{E}_{t}\left[\Gamma_{T} \xi+\int_{0}^{T} \Gamma_{s} P_{s} d s\right]=\Gamma_{t} Y_{t}+\int_{0}^{t} \Gamma_{s} P_{s} d s .
$$

Therefore,

$$
Y_{t}=\Gamma_{t}^{-1}\left(Y_{0}+\int_{0}^{t} \Gamma_{s}\left(Y_{s} R_{s}^{T}+Z_{s}\right) d W_{s}-\int_{0}^{t} \Gamma_{s} P_{s} d s\right)
$$

then one obtains

$$
d Y_{t}=-\left(P_{t}+Y_{t} Q_{t}+Z_{t} R_{t}\right) d t+Z_{t} d W_{t}, \quad Y_{T}=\xi
$$


In particular, (3.6) holds, from which it can be seen that $M_{t}=\int_{0}^{t} Z_{s} d \tilde{W}_{s}$ is a bounded $\tilde{\mathbb{P}}_{-}$ martingale. Since $\tilde{\mathbb{E}}_{\tau} \int_{\tau}^{T}|Z|_{s}^{2} d s=\tilde{\mathbb{E}}_{\tau}\left(M_{T}-M_{\tau}\right)^{2}$, this shows that $Z$ is in $\mathbb{H}^{\mathrm{BMO}}\left(\mathbb{R}^{d \times n}\right)$ with respect to $\tilde{\mathbb{P}}$ and hence, also with respect to $\mathbb{P}$. So we have shown that for a given solution $(U, V) \in \mathbb{S}^{\infty}(\mathbb{R}) \times \mathbb{H}^{\mathrm{BMO}}\left(\mathbb{R}^{1 \times n}\right)$ of the one-dimensional BSDE (3.4), the linear BSDE (3.5) has a unique solution $(Y, Z) \in \mathbb{S}^{\infty}\left(\mathbb{R}^{d}\right) \times \mathbb{H}^{\mathrm{BMO}}\left(\mathbb{R}^{d \times n}\right)$. $\left(a^{T} Y, a^{T} Z\right)$ solves the one-dimensional linear BSDE

$$
\tilde{U}_{t}=a^{T} \xi+\int_{t}^{T}\left(a^{T} P_{s}+\tilde{U}_{s} Q_{s}+\tilde{V}_{s} R_{s}\right) d s-\int_{t}^{T} \tilde{V}_{s} d W_{s}
$$

which, like (3.5), can be shown to have a unique solution in $\mathbb{S}^{\infty}(\mathbb{R}) \times \mathbb{H}^{\mathrm{BMO}}\left(\mathbb{R}^{1 \times n}\right)$. It follows that $\left(a^{T} Y, a^{T} Z\right)=(U, V)$, from which one obtains that $(Y, Z)$ solves the original BSDE (3.2).

If the additional condition (3.3) holds, one obtains from Theorem 2.6 in Kobylanski (2000) that the one-dimensional BSDE (3.4) admits only one solution $(U, V)$ in $\mathbb{S}^{\infty}(\mathbb{R}) \times \mathbb{H}^{\mathrm{BMO}}\left(\mathbb{R}^{1 \times n}\right)$, and it follows that (3.2) has a unique solution $(Y, Z)$ in $\mathbb{S}^{\infty}\left(\mathbb{R}^{d}\right) \times \mathbb{H}^{\mathrm{BMO}}\left(\mathbb{R}^{d \times n}\right)$.

\section{Subquadratic BSDEs}

In the case where the BSDE (1.1) is not Markovian or projectable, we assume the driver $f(s, y, z)$ to be of strictly subquadratic growth in $z$. For constants $C, C_{i} \in \mathbb{R}_{+}, \varepsilon \in(0,1)$ and a nondecreasing function $\rho: \mathbb{R}_{+} \rightarrow \mathbb{R}_{+}$, consider the following conditions:

(B1) $\xi$ is a $d$-dimensional $\mathcal{F}_{T}$-measurable random vector satisfying $|\xi| \leq C$

(B2) $|f(s, y, z)| \leq C\left(1+|y|+\rho(|y|)|z|^{2-\varepsilon}\right)$

(B3) $\left|f(t, y, z)-f\left(t, y^{\prime}, z^{\prime}\right)\right| \leq \rho\left(|y| \vee\left|y^{\prime}\right|\right)\left(\left|y-y^{\prime}\right|+\left(1+\left(|z| \vee\left|z^{\prime}\right|\right)^{1-\varepsilon}\right)\left|z-z^{\prime}\right|\right)$

(B4) $f(s, y, z)=F(s, y, z)+G(s, y, z)$ and

$$
y^{T} F(s, y, z) \leq C|y|(1+|y|+|z|), \quad y^{T} G(s, y, z) \leq\left|y^{T} z\right| \rho(|y|)|z| .
$$

The main result of this section is the following

Theorem 4.1. A BSDE

$$
Y_{t}=\xi+\int_{t}^{T} f\left(s, Y_{s}, Z_{s}\right) d s-\int_{t}^{T} Z_{s} d W_{s}
$$

satisfying (B1)-(B4) has a unique solution $(Y, Z)$ in $\mathbb{S}^{\infty}\left(\mathbb{R}^{d}\right) \times \mathbb{H}^{\mathrm{BMO}}\left(\mathbb{R}^{d \times n}\right)$, and

$$
\left|Y_{t}\right| \leq(C+1) \exp \left(\frac{(C+1)^{2}}{2}(T-t)\right)
$$


We prove Theorem 4.1 by first showing that the BSDE (4.1) has a unique solution for short time intervals and then constructing a solution on $[0, T]$ recursively backwards in time.

For small $h>0$ we use Banach's fixed point theorem to prove the existence and uniqueness of a solution on $[T-h, T]$. For $R \in \mathbb{R}_{+}$, define

$$
\mathcal{B}_{R}:=\left\{(Y, Z) \in \mathbb{S}_{[T-h, T]}^{\infty}\left(\mathbb{R}^{d}\right) \times \mathbb{H}_{[T-h, T]}^{\mathrm{BMO}}\left(\mathbb{R}^{d \times n}\right):\|Y\|_{\mathbb{S}_{[T-h, T]}^{\infty}} \leq R,\|Z\|_{\mathrm{BMO}_{[T-h, T]}} \leq R\right\} .
$$

Lemma 4.2. Assume that $R \geq 3 C$ and (B1)-(B3) hold. Then there exists a constant $\delta>0$ only depending on $C, R$ and $\rho(R)$ such that for all $h \in(0, \delta]$, the BSDE (4.1) has a unique solution $(Y, Z) \in \mathcal{B}_{R}$ on $[T-h, T]$.

Proof. In the whole proof we assume $t \in[T-h, T]$ and treat $\rho=\rho(R)$ as a constant. This is possible because $Y$ will turn out to be bounded by $R$. For $(y, z) \in \mathcal{B}_{R}$, define $\phi(y, z):=(Y, Z)$, where

$$
Y_{t}=\mathbb{E}_{t}\left(\xi+\int_{t}^{T} f\left(s, y_{s}, z_{s}\right) d s\right), \quad T-h \leq t \leq T,
$$

and $Z$ is the unique $\mathbb{R}^{d \times n}$-valued predictable process satisfying

$$
\int_{T-h}^{T} Z_{s} d W_{s}=\xi+\int_{T-h}^{T} f\left(s, y_{s}, z_{s}\right) d s-\mathbb{E}_{T-h}\left(\xi+\int_{T-h}^{T} f\left(s, y_{s}, z_{s}\right) d s\right) .
$$

Then

$$
\left|Y_{t}\right| \leq C+\mathbb{E}_{t} \int_{t}^{T}\left|f\left(s, y_{s}, z_{s}\right)\right| d s, \quad T-h \leq t \leq T
$$

and if $\tau$ is a stopping time with values in $[T-h, T]$, it follows from (B2) and Hölder's inequality that

$$
\begin{aligned}
& \mathbb{E}_{\tau} \int_{\tau}^{T}\left|f\left(s, y_{s}, z_{s}\right)\right| d s \leq C \mathbb{E}_{\tau} \int_{\tau}^{T}\left(1+\left|y_{s}\right|+\rho\left|z_{s}\right|^{2-\varepsilon}\right) d s \\
\leq & C h\left(1+\|y\|_{\mathbb{S}_{[T-h, T]}^{\infty}}\right)+C \rho \mathbb{E}_{\tau} \int_{\tau}^{T}\left|z_{s}\right|^{2-\varepsilon} d s \leq C h\left(1+\|y\|_{\mathbb{S}_{[T-h, T]}^{\infty}}\right)+C \rho h^{\varepsilon / 2}\|z\|_{\mathrm{BMO}_{[\mathrm{T}-\mathrm{h}, \mathrm{T}]}}^{2-\varepsilon} \\
\leq & C h(1+R)+C \rho h^{\varepsilon / 2} R^{2-\varepsilon} .
\end{aligned}
$$

Choose $h>0$ so small that $C h(1+R)+C \rho h^{\varepsilon / 2} R^{2-\varepsilon} \leq C$. Then

$$
\mathbb{E}_{\tau} \int_{\tau}^{T}\left|f\left(s, y_{s}, z_{s}\right)\right| d s \leq C \quad \text { and } \quad\|Y\|_{\mathbb{S}_{[T-h, T]}^{\infty}} \leq 2 C \leq R
$$

On the other hand, it follows from the definition of $(Y, Z)$ that

$$
Y_{t}=\xi+\int_{t}^{T} f\left(s, y_{s}, z_{s}\right) d s-\int_{t}^{T} Z_{s} d W_{s}, \quad T-h \leq t \leq T
$$


So one obtains from Itô's formula,

$$
\left|Y_{\tau}\right|^{2}+\int_{\tau}^{T}\left|Z_{s}\right|^{2} d s=|\xi|^{2}+2 \int_{\tau}^{T} Y_{s}^{T} f\left(s, y_{s}, z_{s}\right) d s-2 \int_{\tau}^{T} Y_{s}^{T} Z_{s} d W_{s}
$$

Since $(y, z) \in \mathbb{S}_{[T-h, T]}^{\infty}\left(\mathbb{R}^{d}\right) \times \mathbb{H}_{[T-h, T]}^{\mathrm{BMO}}\left(\mathbb{R}^{d \times n}\right)$, it follows from (B1) and (B2) that $\xi+\int_{T-h}^{T} f\left(s, y_{s}, z_{s}\right) d s$ is $p$-integrable for some $p>1$. By Doob's maximal $L^{p}$-inequality, $\sup _{T-h \leq t \leq T}\left|\int_{T-h}^{t} Z_{s} d W_{s}\right|$ is also $p$-integrable. So one obtains from the Burkholder-Davis-Gundy inequality that $\int_{T-h}^{T}\left|Y_{s}^{T} Z_{s}\right|^{2} d s$ is $p / 2$-integrable and $\sup _{T-h \leq t \leq T}\left|\int_{T-h}^{t} Y_{s}^{T} Z_{s} d W_{s}\right|$ is $p$-integrable. This implies that the local martingale $\int_{T-h}^{t} Y_{s}^{T} Z_{s} d W_{s}$ is a true martingale. Therefore,

$$
\mathbb{E}_{\tau} \int_{\tau}^{T}\left|Z_{s}\right|^{2} d s \leq C^{2}+2\|Y\|_{\mathbb{S}_{[T-h, T]}^{\infty}} \mathbb{E}_{\tau} \int_{\tau}^{T}\left|f\left(s, y_{s}, z_{s}\right)\right| d s \leq 5 C^{2},
$$

which shows that $\|Z\|_{\mathrm{BMO}_{[T-h, T]}} \leq 3 C \leq R$. So $\phi$ maps $\mathcal{B}_{R}$ into itself.

Next, we show that $\phi$ is a contraction on $\mathcal{B}_{R}$. Choose $(y, z),\left(y^{\prime}, z^{\prime}\right) \in \mathcal{B}_{R}$ and denote $(Y, Z)=$ $\phi(y, z),\left(Y^{\prime}, Z^{\prime}\right)=\phi\left(y^{\prime}, z^{\prime}\right), \Delta y=y-y^{\prime}, \Delta z=z-z^{\prime}, \Delta Y=Y-Y^{\prime}$ and $\Delta Z=Z-Z^{\prime}$. Then,

$$
\Delta Y_{t}=\int_{t}^{T}\left(f\left(s, y_{s}, z_{s}\right)-f\left(s, y_{s}^{\prime}, z_{s}^{\prime}\right)\right) d s-\int_{t}^{T} \Delta Z_{s} d W_{s}
$$

and by Itô's formula,

$$
\left|\Delta Y_{\tau}\right|^{2}+\int_{\tau}^{T}\left|\Delta Z_{s}\right|^{2} d s=2 \int_{\tau}^{T} \Delta Y_{s}^{T}\left(f\left(s, y_{s}, z_{s}\right)-f\left(s, y_{s}^{\prime}, z_{s}^{\prime}\right)\right) d s-2 \int_{\tau}^{T} \Delta Y_{s}^{T} \Delta Z_{s} d W_{s} .
$$

It follows from (B3) that

$$
\begin{aligned}
& \mathbb{E}_{\tau} \int_{\tau}^{T}\left|f\left(s, y_{s}, z_{s}\right)-f\left(s, y_{s}^{\prime}, z_{s}^{\prime}\right)\right| d s \leq \mathbb{E}_{\tau} \int_{\tau}^{T} \rho\left(\left|\Delta y_{s}\right|+\left(1+\left(\left|z_{s}\right| \vee\left|z_{s}^{\prime}\right|\right)^{1-\varepsilon}\right)\left|\Delta z_{s}\right|\right) d s \\
& \leq \rho h\|\Delta y\|_{\mathbb{S}_{[T-h, T]}^{\infty}}+\rho \sqrt{\mathbb{E}_{\tau} \int_{\tau}^{T}\left(1+\left(\left|z_{s}\right| \vee\left|z_{s}^{\prime}\right|\right)^{1-\varepsilon}\right)^{2} d s} \sqrt{\mathbb{E}_{\tau} \int_{\tau}^{T}\left|\Delta z_{s}\right|^{2} d s} \\
& \leq \rho h\|\Delta y\|_{\mathbb{S}_{[T-h, T]}^{\infty}}+\rho \sqrt{2\left(h+\mathbb{E}_{\tau} \int_{\tau}^{T}\left(\left|z_{s}\right|+\left|z_{s}^{\prime}\right|\right)^{2-2 \varepsilon} d s\right)}\|\Delta z\|_{\mathrm{BMO}_{[T-h, T]}} \\
& \leq \rho h\|\Delta y\|_{\mathbb{S}_{[T-h, T]}^{\infty}}+\rho \sqrt{2\left(h+h^{\varepsilon}(2 R)^{2-2 \varepsilon}\right)}\|\Delta z\|_{\mathrm{BMO}_{[T-h, T]}} .
\end{aligned}
$$

So for $h>0$ small enough, one has

$$
\begin{aligned}
& \mathbb{E}_{\tau} \int_{\tau}^{T}\left|f\left(s, y_{s}, z_{s}\right)-f\left(s, y_{s}^{\prime}, z_{s}^{\prime}\right)\right| d s \leq \frac{1}{4}\left(\|\Delta y\|_{\mathbb{S}_{[T-h, T]}^{\infty}}+\|\Delta z\|_{\mathrm{BMO}_{[T-h, T]}}\right) \\
& \leq \frac{1}{2}\left(\|\Delta y\|_{\mathbb{S}_{[T-h, T]}^{\infty}} \vee\|\Delta z\|_{\mathrm{BMO}_{[T-h, T]}}\right) .
\end{aligned}
$$


Since $\int_{T-h}^{t} \Delta Z_{s} d W_{s}$ and $\int_{T-h}^{t} \Delta Y_{s}^{T} \Delta Z_{s} d W_{s}$ are martingales, one obtains from (4.3),

$$
\|\Delta Y\|_{\mathbb{S}_{[T-h, T]}^{\infty}} \leq \frac{1}{2}\left(\|\Delta y\|_{\mathbb{S}_{[T-h, T]}^{\infty}} \vee\|\Delta z\|_{\mathrm{BMO}_{[T-h, T]}}\right)
$$

and from (4.4),

$$
\begin{aligned}
& \|\Delta Z\|_{\mathrm{BMO}_{[T-h, T]}}^{2} \leq 2\|\Delta Y\|_{\mathbb{S}_{[T-h, T]}^{\infty}} \sup _{\tau} \mathbb{E}_{\tau} \int_{\tau}^{T}\left|f\left(s, y_{s}, z_{s}\right)-f\left(s, y_{s}^{\prime}, z_{s}^{\prime}\right)\right| d s \\
& \leq \frac{1}{2}\left(\|\Delta y\|_{\mathbb{S}_{[T-h, T]}^{\infty}} \vee\|\Delta z\|_{\mathrm{BMO}_{[T-h, T]}}\right)^{2} .
\end{aligned}
$$

This shows that there exists a $\delta>0$ only depending on $C, R$ and $\rho(R)$ such that for $h \in(0, \delta]$, $\phi$ is a contraction on $\mathcal{B}_{R}$, from which it follows that on $[T-h, T]$, (4.1) has a unique solution in $\mathcal{B}_{R}$.

In the next step we show that under the additional condition (B4), the solution of Lemma 4.2 satisfies

$$
\left|Y_{t}\right| \leq(C+1) \exp \left(\frac{(C+1)^{2}}{2}(T-t)\right) .
$$

This allows us to construct a solution on the whole time interval $[0, T]$ recursively.

Lemma 4.3. Assume (B1) and (B4) hold, and for some $h>0$, the BSDE (4.1) has a solution $(Y, Z) \in \mathbb{S}_{[T-h, T]}^{\infty}\left(\mathbb{R}^{d}\right) \times \mathbb{H}_{[T-h, T]}^{\mathrm{BMO}}\left(\mathbb{R}^{d \times n}\right)$. Then

$$
\left|Y_{t}\right| \leq(C+1) \exp \left(\frac{(C+1)^{2}}{2}(T-t)\right)
$$

for $t \in[T-h, T]$.

Proof. Fix $a \in \mathbb{R}_{+}$and note that for all $i=1, \ldots, d$, one obtains from Itô's formula,

$$
\begin{aligned}
& e^{a t}\left|Y_{t}\right|^{2}=e^{a T}|\xi|^{2}+2 \int_{t}^{T} e^{a s}\left(Y_{s}^{T} f\left(s, Y_{s}, Z_{s}\right)-\frac{a}{2}\left|Y_{s}\right|^{2}-\frac{1}{2}\left|Z_{s}\right|^{2}\right) d s-2 \int_{t}^{T} e^{a s} Y_{s}^{T} Z_{s} d W_{s} \\
& =e^{a T}|\xi|^{2}+2 \int_{t}^{T} e^{a s}\left(Y_{s}^{T} f\left(s, Y_{s}, Z_{s}\right)-\frac{a}{2}\left|Y_{s}\right|^{2}-\frac{1}{2}\left|Z_{s}\right|^{2}-Y_{s}^{T} Z_{s}\left(\rho\left(\left|Y_{s}\right|\right)\left|Z_{s}\right| \frac{Y_{s}^{T} Z_{s}}{\left|Y_{s}^{T} Z_{s}\right|}\right)^{T}\right) d s \\
& \quad-2 \int_{t}^{T} e^{a s} Y_{s}^{T} Z_{s} d \tilde{W}_{s},
\end{aligned}
$$

where

$$
\tilde{W}_{s}=W_{s}-\int_{0}^{t}\left(\rho\left(\left|Y_{s}\right|\right)\left|Z_{s}\right| \frac{Y_{s}^{T} Z_{s}}{\left|Y_{s}^{T} Z_{s}\right|}\right)^{T} d s
$$


is a Brownian motion under the equivalent probability measure

$$
\tilde{\mathbb{P}}:=\exp \left(\int_{0}^{T} \rho\left(\left|Y_{s}\right|\right)\left|Z_{s}\right| \frac{Y_{s}^{T} Z_{s}}{\left|Y_{s}^{T} Z_{s}\right|} d W_{s}-\frac{1}{2} \int_{0}^{T}\left(\rho\left(\left|Y_{s}\right|\right)\left|Z_{s}\right|\right)^{2} d s\right) \cdot \mathbb{P} .
$$

Denote by $\tilde{\mathbb{E}}$ the expectation with respect to $\tilde{\mathbb{P}}$ and notice that

$$
\begin{aligned}
& Y_{s}^{T} f\left(s, Y_{s}, Z_{s}\right)-\frac{a}{2}\left|Y_{s}\right|^{2}-\frac{1}{2}\left|Z_{s}\right|^{2}-Y_{s}^{T} Z_{s}\left(\rho\left(\left|Y_{s}\right|\right)\left|Z_{s}\right| \frac{Y_{s}^{T} Z_{s}}{\left|Y_{s}^{T} Z_{s}\right|}\right)^{T} \\
& =Y_{s}^{T} F\left(s, Y_{s}, Z_{s}\right)+Y_{s}^{T} G\left(s, Y_{s}, Z_{s}\right)-\frac{a}{2}\left|Y_{s}\right|^{2}-\frac{1}{2}\left|Z_{s}\right|^{2}-\left|Y_{s}^{T} Z_{s}\right| \rho\left(\left|Y_{s}\right|\right)\left|Z_{s}\right| \\
& \leq C\left|Y_{s}\right|\left(1+\left|Y_{s}\right|+\left|Z_{s}\right|\right)-\frac{a}{2}\left|Y_{s}\right|^{2}-\frac{1}{2}\left|Z_{s}\right|^{2} \\
& \leq \frac{C^{2}}{2}+\frac{1}{2}\left((C+1)^{2}-a\right)\left|Y_{s}\right|^{2} .
\end{aligned}
$$

So for $a=(C+1)^{2}$, one obtains

$$
e^{a t}\left|Y_{t}\right|^{2} \leq C^{2} e^{a T}+C^{2} \int_{t}^{T} e^{a s} d s-2 \int_{t}^{T} e^{a s} Y_{s}^{T} Z_{s} d \tilde{W}_{s},
$$

which by applying $\tilde{\mathbb{E}}_{t}$, gives $\left|Y_{t}\right|^{2} \leq C^{2} e^{a(T-t)}(a+1) / a$, and therefore,

$$
\left|Y_{t}\right| \leq C \exp \left(\frac{(C+1)^{2}}{2}(T-t)\right) \sqrt{\frac{C^{2}+1}{C^{2}}} \leq(C+1) \exp \left(\frac{(C+1)^{2}}{2}(T-t)\right) \text {. }
$$

\section{Proof of Theorem 4.1}

Choose $h>0$ such that $T / h \in \mathbb{N}$ and the mapping $\phi$ from the proof of Lemma 4.2 is a contraction when $C$ is replaced by $(C+1) \exp \left((C+1)^{2} T / 2\right)$. Then (4.1) has a unique solution $\left(Y^{(1)}, Z^{(1)}\right) \in \mathbb{S}_{[T-h, T]}^{\infty}\left(\mathbb{R}^{d}\right) \times \mathbb{H}_{[T-h, T]}^{\mathrm{BMO}}\left(\mathbb{R}^{d \times n}\right)$ on $[T-h, T]$. By Lemma 4.3, one has $\left|Y_{t}^{(1)}\right| \leq$ $\varphi(t):=(C+1) \exp \left((C+1)^{2}(T-t) / 2\right)$. Another application of Lemma 4.2 yields that on $[T-$ $2 h, T-h]$, the BSDE (4.1) with terminal condition $Y_{T-h}^{(1)}$ has a unique solution $\left(Y^{(2)}, Z^{(2)}\right) \in$ $\mathbb{S}_{[T-2 h, T-h]}^{\infty}\left(\mathbb{R}^{d}\right) \times \mathbb{H}_{[T-2 h, T-h]}^{\mathrm{BMO}}\left(\mathbb{R}^{d \times n}\right)$. Pasting together the two solutions gives a solution $(Y, Z) \in$ $\mathbb{S}_{[T-2 h, T]}^{\infty}\left(\mathbb{R}^{d}\right) \times \mathbb{H}_{[T-2 h, T]}^{\mathrm{BMO}}\left(\mathbb{R}^{d \times n}\right)$ on $[T-2 h, T]$. By Lemma 4.3, it satisfies $\left|Y_{t}\right| \leq \varphi(t)$. Continuing like this yields a unique solution $(Y, Z) \in \mathbb{S}^{\infty}\left(\mathbb{R}^{d}\right) \times \mathbb{H}^{\mathrm{BMO}}\left(\mathbb{R}^{d \times n}\right)$ on $[0, T]$, which by Lemma 4.3, satisfies $\left|Y_{t}\right| \leq \varphi(t)$.

\section{Acknowledgements}

We thank Peng Luo and two anonymous referees for helpful comments. 


\section{References}

[1] Ankirchner, S., Imkeller, P., Popier, A. (2009). On measure solutions of backward stochastic differential equations. Stoch. Proc. Appl. 119(9), 2744-2772.

[2] Bahlali, K. (2001). Backward stochastic differential equations with locally Lipschitz coefficient. C. R. Acad. Sci. Paris Sér. I Math. 333(5), 481-486.

[3] Bahlali, K., Essaky, E.H., Hassani, M. (2010). p-integrable solutions to multidimensional BSDEs and degenerate systems of PDEs with logarithmic nonlinearities. Preprint arXiv:1007.2388, http://arxiv.org/abs/1007.2388.

[4] Briand, P., Elie, R. (2013). A simple constructive approach to quadratic BSDEs with or without delay. Stoch. Proc. Appl. 123(8), 2921-2939.

[5] Cheridito, P., Nam, K. (2014). BSDEs with terminal conditions that have bounded Malliavin derivative. J. Funct. Analysis, 266(3), 1257-1285.

[6] Chitashvili, R., Mania, M. (1996). Generalized Itô formula and derivation of Bellman's equation. Stochastic processes and related topics (Siegmundsberg, 1994), 1-21, Stochastics Monograph, 10, Gordon and Breach, Yverdon.

[7] Delarue, F. (2002). On the existence and uniqueness of solutions to FBSDEs in a nondegenerate case. Stoch. Proc. Appl. 99(2), 209-286.

[8] El-Karoui, N., Peng, S., Quenez, M.C. (1997). Backward stochastic differential equations in finance. Math. Finance, 7(1), 1-71.

[9] Frei, C., Reis, Dos, G. (2011). A financial market with interacting investors: does an equilibrium exist? Math. Fin. Economics, 4(3), 161-182.

[10] Hu, Y., Peng, S. (2006). On the comparison theorem for multidimensional BSDEs. C. R. Math. Acad. Sci. Paris 343(2), 135-140.

[11] Kazamaki, N. (1994). Continuous exponential martingales and BMO, Lecture Notes in Mathematics, 1579. Springer-Verlag, Berlin.

[12] Kobylanski, M. (2000). Backward stochastic differential equations and partial differential equations with quadratic growth. Ann. Prob. 28(2), 558-602.

[13] Liang, G., Lionnet, A., Qian, Z. (2010). On Girsanov's transform for backward stochastic differential equations. Preprint arXiv:1011.3228, http://arxiv.org/abs/1011.3228.

[14] Mania, M., Schweizer, M. (2005). Dynamic exponential utility indifference valuation. Ann. Appl. Prob. 15(3), 2113-2143. 
[15] Pardoux, E. (1999). BSDEs, weak convergence and homogenization of semilinear PDEs. Nonlinear analysis, differential equations and control (Montreal, QC, 1998), 503-549.

[16] Pardoux, E.,Peng, S. (1990). Adapted solution of a backward stochastic differential equation, Systems Contr. Lett. 14(1), 55-61.

[17] Pardoux, E., Tang, S. (1999). Forward-backward stochastic differential equations and quasilinear parabolic PDEs. Prob. Th. Rel. Fields 114(2), 123-150.

[18] Peng, S. (1999). Open problems on backward stochastic differential equations. Control of distributed parameter and stochastic systems (Hangzhou, 1998), 265-273, Kluwer Acad. Publ., Boston, MA.

[19] Tevzadze, R. (2008). Solvability of backward stochastic differential equations with quadratic growth. Stoch. Proc. Appl. 118(3), 503-515. 